\title{
Methanofullerene-coated tetrabenzoporphyrin organic field-effect transistors
}

\author{
Patrick B. Shea and Jerzy Kanicki ${ }^{\text {a) }}$ \\ Organic and Molecular Electronics Laboratory, Department of Electrical Engineering and \\ Computer Science, The University of Michigan, Ann Arbor, Michigan 48109 \\ Yong Cao \\ Institute of Polymer Optoelectronic Materials and Devices, College of Materials Science, \\ South China University of Technology, Building 25, Room 4001, Wushan Rd., Guangzhou, China \\ Noboru Ono \\ Department of Chemistry in the Faculty of Science, Ehime University, Bunkyo-cho 2-5, \\ Matsuyama 790-8577, Japan
}

(Received 20 May 2005; accepted 30 August 2005; published online 19 October 2005)

\begin{abstract}
Organic field-effect transistors (OFETs) using a solution-processable form of the organic semiconductor tetrabenzoporphyrin were fabricated with a top coating of a soluble $n$-type organic semiconductor. The top coating was found to extend the lifetime of the device in that the field-effect mobility, subthreshold slope, and OFF-state current were maintained at or near their as-fabricated states. Oxygen doping by extended air exposure was effectively slowed by the electron-accepting coating layer. Coated devices also display a transfer characteristic indicative of a parasitic latch-up transistor formed at the back channel of the OFETs. () 2005 American Institute of Physics.
\end{abstract}

[DOI: $10.1063 / 1.2115077]$

Organic field-effect transistors (OFETs) enjoy a wide and intense research effort as potential devices for low-cost flexible electronics. ${ }^{1-3}$ Solution processable organic semiconductors, such as polymers or small molecules, typically fit the bill for such applications. However, ease of processing often comes at the cost of durability, in the sense that many organic semiconductors are very sensitive to ambient effects or postdeposition processing, namely the effects of absorbing oxygen, ${ }^{4}$ or water, ${ }^{5}$ or exposure to light. ${ }^{6}$ In the case of processing following thin-film deposition processing, Yoshida et $\mathrm{al}^{7}$ coated vacuum-deposited pentacene with vacuumdeposited layers of $n$ - and $p$-type organic semiconductors, while Rost et $a l^{8-10}$ utilized an evaporated bilayer $n$ - and $p$-type structure to achieve ambipolar and light-emitting OFET behavior. Several works have examined tetrabenzoporphyrin (TBP), ${ }^{11-13}$ a high-performance organic semiconductor whose simplicity and customizability offer great promise for organic semiconductors. After conversion from a precursor thin-film, TBP becomes insoluble, making it ideal for postprocessing steps such as metallization and encapsulation. In this work, we examine the effect of encapsulation with a soluble methanofullerene on staggered electrode OFETs fabricated using solution-processed TBP.

OFETs were fabricated using staggered source and drain electrodes and an unpatterned gate. Highly doped crystalline silicon wafers with a $100 \mathrm{~nm}$ thermal oxide were used as the substrate, unpatterned gate electrode, and gate insulator. Before organic thin-film deposition, substrates were cleaned by washing in acetone and isopropyl alcohol, followed by a 20 min exposure to ultraviolet (UV)/ozone. The substrates were then soaked in reagent-grade ethanol for $20 \mathrm{~min}$ and dried with nitrogen gas. A $0.7 \%$ weight precursor solution was prepared by dissolving 1,4:8,11:15,18-22,25-

\footnotetext{
a) Author to whom correspondence should be addressed; electronic mail:
} kanicki@eecs.umich.edu
tetraethano-29H,31H-tetrabenzo[b,g,l,q]porphine (CP) in chloroform, spun-cast onto the substrates, then thermally annealed in nitrogen at $210{ }^{\circ} \mathrm{C}$ for $10 \mathrm{~min}$ to convert $\mathrm{CP}$ to TBP. Source and drain electrodes were then formed by thermally evaporating $60 \mathrm{~nm}$ of Au through a stencil mask. A $1 \%$ weight solution of $[6,6]$-phenyl- $\mathrm{C}_{61}$ butyric acid methyl ester (PCBM) (Ref. 14) was also produced in chloroform. After initial electrical testing of the TBP OFETs, the PCBM solution was spun-cast on top of the devices and thermally annealed in vacuum at $90{ }^{\circ} \mathrm{C}$ to drive off the solvent. To examine a device's transfer characteristics, the gate-source bias $\left(V_{\mathrm{GS}}\right)$ was swept from the ON state $\left(V_{\mathrm{GS}} \gg V_{T}\right)$ to the OFF state $\left(V_{\mathrm{GS}} \ll V_{T}\right)$, and back. To examine the output characteristic, $V_{\mathrm{GS}}$ was stepped from ON to OFF, while during each step the drain-source bias, $V_{\mathrm{DS}}$, was swept from $0 \mathrm{~V}$ to $-40 \mathrm{~V}$. Between tests, devices were stored in a dry nitrogen atmosphere $\left(<100 \mathrm{ppm} \mathrm{O}_{2},<1 \mathrm{ppm}_{2} \mathrm{O}\right)$. We also coated TBP OFETs with the $p$-type air-stable organic semiconducting polymer F8T2 ${ }^{6}$ While the long-term air exposure effects of the F8T2 coating are still being studied, in the short-term F8T2-coated TBP OFETs exhibit a reduced oxidation effect. However, whereas PCBM-coated TBP OFETs exhibit a consistent change in $\mu_{\mathrm{FE}}, V_{T}$, and $I_{\mathrm{OFF}}$, F8T2-coated TBP OFETs produce inconsistent results with regards to the same parameters. Furthermore, many $p$-type polymers are permanently effected by air exposure, whereas PCBM-as a coating here-required only low-temperature vacuum annealing to reverse air exposure effects.

Output and transfer characteristics immediately before and after coating are shown in Figs. 1 and 2, respectively. Distinct linear and saturation regimes are observed in Fig. 1 for both cases; no notable change in the contact resistance is observed. Ambipolar behavior is not observed for coated devices, either before or after exposure to air. Interestingly enough, the output characteristics for the coated OFETs display an enhancement in $I_{D}$ as compared to the uncoated de- 


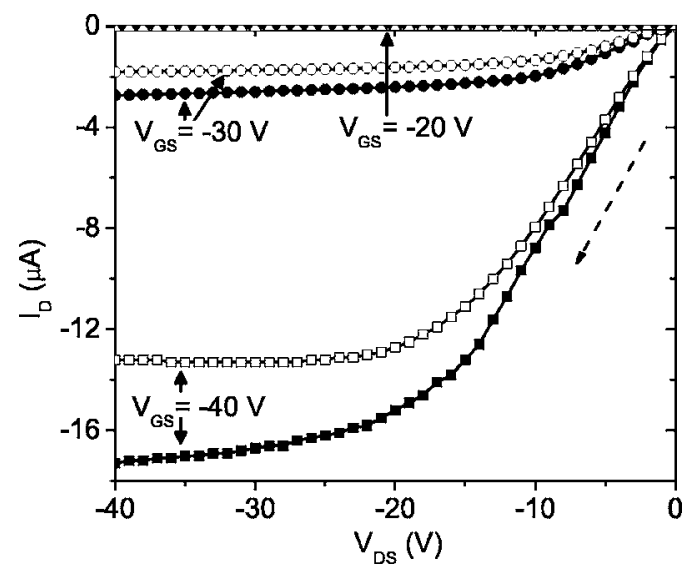

FIG. 1. Output characteristics of a TBP-PCBM OFET immediately before and after coating. Open markers indicate the uncoated devices, while closed markers indicate the coated devices. The dashed arrow indicates the direction of measurement.

vices. The transfer characteristics in the linear regime (Fig. $2)$, however, indicate that the threshold voltage, $V_{T}(\cong$ $-21 \mathrm{~V} \pm 4$ ), becomes more negative after the TBP OFETs are coated with PCBM, which is known to be an electron acceptor. ${ }^{14}$ Shifts in $V_{T}$ from $-1 \mathrm{~V}$ to $-4 \mathrm{~V}$ have been observed in the linear regime. Negligible differences are observed in the field-effect mobility, $\mu_{\mathrm{FE}}(\cong 5$ $\left.\times 10^{-3} \mathrm{~cm}^{2} / \mathrm{V}-\mathrm{s} \pm 4 \times 10^{-3}\right)$, and the subthreshold slope, $S(\cong 1.8 \mathrm{~V} / \mathrm{dec} \pm 0.90)$. However, coated devices exhibit a small increase in the OFF-state current, from $-50 \mathrm{pA}$ to $-100 \mathrm{pA}$; and a kink is noticeable in the transfer characteristics around $V_{\mathrm{GS}}=-20 \mathrm{~V}$. We believe that this kink, which appears during initial electrical testing of coated devices but disappears after prolonged exposure to air (Fig. 3), results from the formation of a parasitic back-channel latch-up transistor. ${ }^{15}$ As the accumulated charges from the OFET channel discharge in the subthreshold regime, the presence of the latch-up transistor can noticeably effect device operation; the effect is not observed when sweeping the devices from OFF to ON. Our preliminary measurements also indicate that the PCBM coating induces a significant increase in hysteresis in the transfer characteristics, whereas Aramaki et al. ${ }^{12}$ reported that coating with an insulator, such as poly-

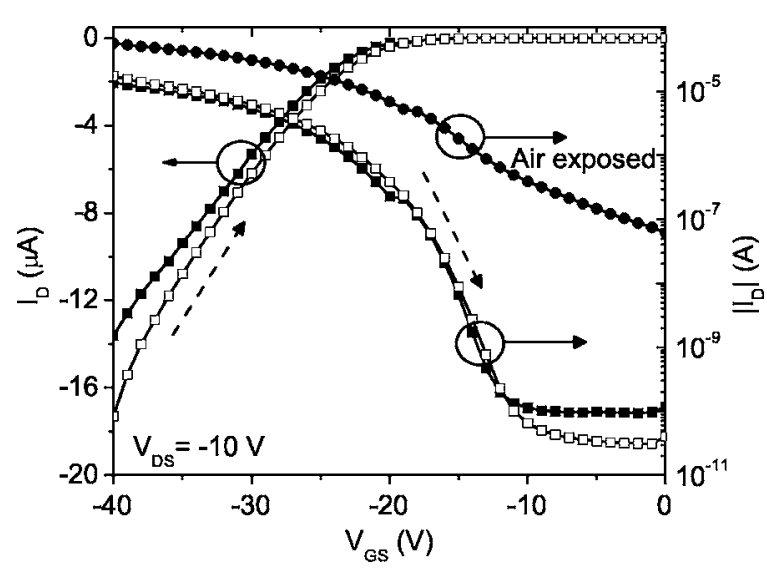

FIG. 2. Linear regime transfer characteristics of a TBP-PCBM OFET immediately before and after coating. Open markers indicate the uncoated devices, while closed markers indicate the coated devices. The dashed arrows indicate the direction of measurement. An uncoated TBP OFET after a 1 week exposure to dry air is also shown.

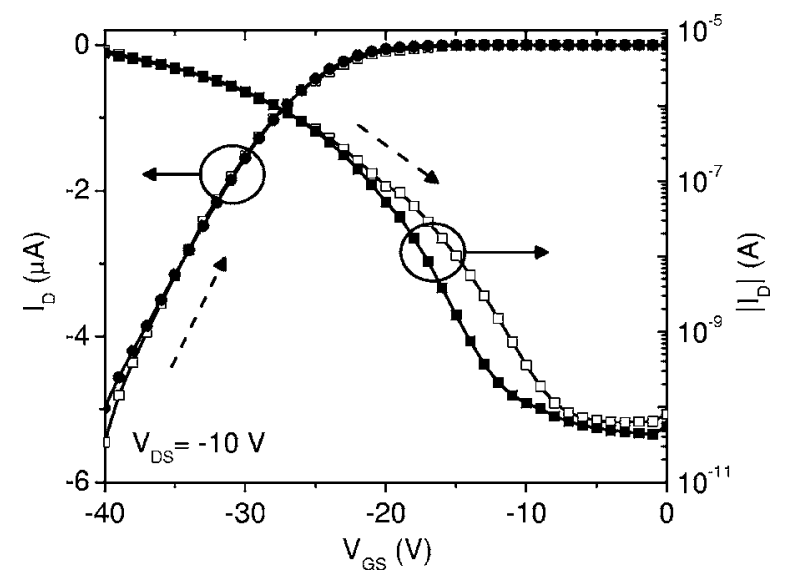

FIG. 3. Linear regime transfer characteristics of a TBP-PCBM OFET immediately after coating, and again 8 months after coating. Open markers indicate just-coated devices, while closed markers indicate 8 month old coated devices. The dashed arrows indicate the direction of measurement.

methylmethacrylate, effectively reduced transfer characteristic hysteresis. The cumulative effect of extended testing in dry air and the low $\mathrm{O}_{2}$ content of the storage environment was observed to effect uncoated OFETs, as shown in Fig. 2. In that case, exposure to air produced an immediate increase in the thin-film conductivity, which manifested itself in an increase in the accumulation regime field-effect mobility, the bulk conductivity, and the subthreshold swing. A similar effect was not observed here for coated devices.

After initial device fabrication and testing, the OFETs were vacuum annealed at $90{ }^{\circ} \mathrm{C}$ and returned to the aforementioned nitrogen environment for 8 months. Devices were then thermally annealed and tested in air to observe the effect of the extended presence of the PCBM coating on OFET behavior. Linear regime transfer characteristics exhibit minimal change after 8 months storage. A slightly lower $S$, as compared to just-coated devices, is observed. To clearly characterize the electrical response of the PCBM-coated TBP OFETs to air, after 8 months time, the devices were vacuum annealed at $90{ }^{\circ} \mathrm{C}$ and tested during an extended exposure to air. A summary display of key linear regime device characteristics is shown in Fig. 4. A trend with exposure to air is observed, with a decrease in the magnitude of $V_{T}$, and increases in $\mu_{\mathrm{FE}}, S$, and $I_{\mathrm{OFF}}$. However, the trend is reversible by thermal vacuum annealing at $90{ }^{\circ} \mathrm{C}$, and is not as marked as for uncoated devices. By comparison, a thermal vacuum anneal above $200{ }^{\circ} \mathrm{C}$ is required to reverse the exposure effect in uncoated TBP OFETs, but even then the subthreshold slope is permanently effected.

Misalignment between the highest occupied and lowest unoccupied molecular orbitals of TBP (Ref. 13) and PCBM (Ref. 14) indicates that a PCBM coating will prevent electrons from entering the TBP film, preventing doping of the TBP that would increase its effective hole density. Indeed, the electrophilicity of fullerenes is well known, ${ }^{16}$ and so the propensity of PCBM to reduce acts as a buffer to the airborne $\mathrm{O}_{2}$. The effect of PCBM on the TBP OFET electrical parameters can also be explained as follows. The increase in $I_{D}$ for $V_{\mathrm{GS}}>-10 \mathrm{~V}$ results from the presence of more electron conducting material near the source and drain electrodes. The shift in $V_{T}$, and the increase in hysteresis, likely result from an increase in positive space charge. $\mathrm{A} \mathrm{C}_{60}$ molecule can accept up to six electrons, and so the PCBM coat- 


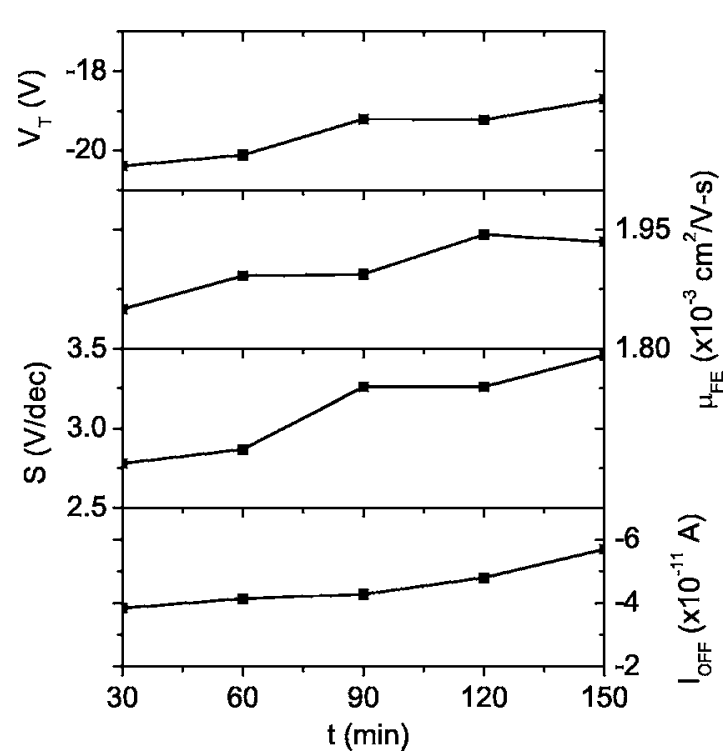

FIG. 4. Dependence of the linear regime device electrical parameters over 150 min exposure to air for the aged devices shown in Fig. 3. Measured values at $t=0 \mathrm{~min}$ are negligibly different from $t=30 \mathrm{~min}$.

ing is eventually permeated. It was reported for packaged PCBM OFETs that exposure to an ambient atmosphere increases $\mu_{\mathrm{FE}}$ and lowers the magnitude of $V_{T}$; the OFF-state current remains the same. ${ }^{17}$ Thus, we can deduce that as the PCBM only reduces so much, oxygen eventually permeates through to the TBP thin film. Either a thicker encapsulation layer, or an film made of an electrically inert material, might be needed to completely stabilize the electrical properties of such devices.

We have studied the effects of coating solutionprocessed tetrabenzoporphyrin OFETs with a solution- processed methanofullerene thin film. Ambipolar transistor operation is not observed for these spun-cast devices, but an enhancement in device lifetime is observed in that exposure to air does not degrade device electrical performance as quickly as it does for uncoated OFETs. Coated transistors exhibit a shift in the threshold voltage, as well as a kink in the transfer characteristics that may indicate the formation of a parasitic latch-up transistor.

${ }^{1}$ G. Horowitz, Adv. Mater. (Weinheim, Ger.) 10, 365 (1998).

${ }^{2}$ C. Dimitrakopoulos and D. Mascaro, IBM J. Res. Dev. 45, 11 (2001).

${ }^{3}$ S. Scheinert and G. Paasch, Phys. Status Solidi A 201, 1263 (2004).

${ }^{4}$ Z. Bao, Adv. Mater. (Weinheim, Ger.) 12, 227 (2000).

${ }^{5}$ Y. Qiu, Y. C. Hu, G. F. Dong, L. D. Wang, J. F. Xie, and Y. N. Ma, Appl. Phys. Lett. 83, 1644 (2003).

${ }^{6}$ M. C. Hamilton, S. Martin, and J. Kanicki, IEEE Trans. Electron Devices 51, 877 (2004).

${ }^{7}$ M. Yoshida, S. Uemura, T. Kodzasa, H. Ushijima, and T. Kamata, Synth. Met. 137, 893 (2003).

${ }^{8}$ C. Rost, D. J. Gundlach, S. Karg, and W. Rieb, Synth. Met. 95, 5782 (2004).

${ }^{9}$ C. Rost, S. Karg, W. Riess, M. A. Loi, M. Murgia, and M. Muccini, Appl. Phys. Lett. 85, 1613 (2004).

${ }^{10}$ C. Rost, S. Karg, W. Riess, M. A. Loi, M. Murgia, and M. Muccini, Synth. Met. 146, 237 (2004).

${ }^{11}$ S. Aramaki, Y. Sakai, and N. Ono, Appl. Phys. Lett. 84, 2085 (2004).

${ }^{12}$ S. Aramaki, Y. Sakai, R. Yoshiyama, K. Sugiyama, N. Ono, and J. Mizuguchi, Proc. SPIE 5522, 27 (2004).

${ }^{13}$ P. B. Shea, A. R. Johnson, J. Kanicki, and N. Ono, IEEE Trans. Electron Devices 52, 1497 (2005).

${ }^{14}$ V. D. Mihailetchi, J. K. van Duren, P. W. Blom, J. C. Hummelen, R. A. Janssen, J. M. Kroon, M. T. Rispens, W. J. H. Verhees, and M. M. Wienk, Adv. Funct. Mater. 13, 43 (2003).

${ }^{15}$ J. D. Plummer, M. D. Deal, and P. B. Griffin, Silicon VLSI Technology (Prentice-Hall, Upper Saddle River, New Jersey, 2000).

${ }^{16} \mathrm{M}$. Dresselhaus, G. Dresselhaus, and P. Eklund, Science of Fullerenes and Carbon Nanotubes (Academic, San Diego, CA, 1996).

${ }^{17}$ C. Waldauf, P. Schilinsky, M. Perisutti, J. Hauch, and C. J. Brabec, Adv. Mater. (Weinheim, Ger.) 15, 2084 (2003). 